\title{
A structured review of different rotary instumentation - a decade long reference analysis
}

\begin{abstract}
The objective of the present work is to carry out a bibliographical review of the last tenyears about rotary instrumentation in endodontics and the different types of files that can be used, searching for a comparative of advantages and disadvantages and even possible indications for each case. File type or procedure. The rotational endodontic system has meant an extraordinary advance in the treatment of ducts of both anterior and posterior teeth. The rotary instrumentation allows a smaller number of limes to be used, giving it a greater conicity that facilitates the cleaning of the duct and its subsequent filling. The aim of this work is to explain the technique of mechanical instrumentation of the root canal, exposing its design, characteristics of composition, form of use and the advantages or disadvantages with respect to other systems.
\end{abstract}

Keywords: duct treatment, rotary instrumentation, nickel chrome files, manual instrumentation, mtwo, $\mathrm{k} 3$, protaper, wave one
Volume 5 Issue I - 2018

\author{
Marcos Moradas Estrada \\ Department of Surgery and Surgical Medical Specialties, \\ University of Oviedo, Spain
}

\begin{abstract}
Correspondence: Marcos Moradas Estrada, Associate professor, Service of Conservative Dentistry and Dental Materials. Department of Surgery and Surgical Medical Specialties, University of Oviedo, Spain, Email marcosmords@gmail.com
\end{abstract}

Received: May 17, 2017 | Published: January 18, 2018

\section{Introduction}

The treatment of the root canals of a tooth has in the last twentyfiveyears a major development and improvement of its technique and procedure, allowing better results with greater control of work and in less time. Hand in hand has come a drastic change with the appearance of new techniques, equipment, materials and instruments, among which the rotary endodontic system stands out.

The history of endodontics has always been marked by the search for faster, safer and more efficient procedures that will keep two common objectives and continue to be the cornerstone of any dental treatment: duct conformation and disinfection. Close and curved root canals pose a challenge, even if the professional has a long experience, given the impossibility of achieving the aforementioned objectives with the certainty of not breaking the instrument or generating an iatrogeny in the tooth. Recently, a new metal alloy, made of nickel titanium (Ni-Ti), has been developed in endodontics thanks to its excellent properties of flexibility, torsion resistance and memory in terms of its shape. This novel system consists of a variety of files made as we say in nickel titanium, which are more flexible than those of traditionally used stainless steel, which avoids or try to avoid fractures inside the duct. This type of rotary instrumentation constitutes or represents the third generation in the improvement and simplification of the treatment of the root canal, being considered a new era accepted and contrasted in the daily activity of the dentist. We call it rotational instrumentation by the type of conformation that is made with instruments capable of rotating $360^{\circ}$ inside the root canal (strawberries and limes), driven by a source of energy (low revolution) that aims to achieve the following objectives:

a. Cleaning and disinfection of necrotic tissue rests, both pulp tissues and dentin walls.

b. Conformation of the intraradicular cavity walls determined by the sealing material.

c. Treatment with the same success rate and predictability for straight and homogeneous ducts, as in the case of curved, abrupt or semi-cystic ducts or requiring retreatment.

Therefore, the development of systems using nickel titanium instruments was an event that revolutionized endodontics as previously known, incorporating a number of conceptual changes in the preparation of the root canal system. These instruments allow to increase the speed and efficiency of the treatment, without posing risk to the patient or professional. This type of instrumentation presents the same indications as classic manual endodontics, changing only in the number of files to be used and in that they are activated at least by a micromotor that at low revolution perform the oscillating movement to permeabilize the conduit, give The length of work and subsequently the disinfection of the walls of the light of the conduit and thus prepare it For subsequent sealing. In addition the so-called 'endodontic motor' allows a greater number of options such as two-way clockwise and counterclockwise instrumentation, determining working length and even allowing different systems or types of files to be used for what has been coined as' rotary endodontics Or mechanized ', to which we have to add a more precise control, constant and with less noise pollution. This procedure or set of procedures is also possible against the dreaded curved ducts, showing interesting and optimal results, with hardly any cases of 'zip' or 'false paths generated in the curved section.

It is again of vital importance, as well as in manual instrumentation, presenting more cases in the rotary, the unexpected fracture of instruments, in many cases without previous visible permanent deformation. Fractures in rotating instruments can occur in two ways: torsional fracture and flexural fracture.

TORSIONAL FRACTURE: This occurs when the tip of the file or any other part of the instrument gets stuck in the duct, while the remaining part is rotated inside the duct.

\section{Fracture by flexion}

They occur due to the fatigue that the material undergoes in root 
canals with small radius of curvature, where the limit of flexibility of the instruments is exceeded, resulting in the cyclic fatigue of the same. Even this fracture is generated by the use itself, in ducts morphologically straight and homogeneous, this is the importance that some authors give to the number of uses of each file. Certain manufacturers establish a maximum number of instruments, but with many gaps that justify the data: if we imagine that the manufacturer stipulates a number of uses, it is equal to the fatigue suffered by the file in a straight and homogeneous channel that in a curved and Abrupt, wear in a duct of a monorradicular tooth is similar to that in a bi or multiradicular tooth, and is similar in anterior tooth as in posterior tooth. Not to mention that we must take into account the maintenance after use that we give the file and how it can favor its wear.

Thus the present work aims to know the general characteristics of the rotational systems, knowing the biomechanical preparation capacity of the root canals in terms of their shape, time of use, fracture and risk of suffering, degree of cleaning and Disinfection before each procedure or type of rotary instrument, regardless of the motor used.

\section{Historical review: how it was achieved}

In 1838 Edward Maynard created the first endodontic instrument starting from the spring of a clock with the aim of cleaning and widening the root canal. This technical principle advocated by Maynard persisted until recently, because, in order to widen a root canal properly, to the file $\mathrm{K} \mathrm{n}^{\circ} 25$ and pushing with that of the number 10 , it was necessary approximately 1200 movements of introductory pressure and oscillating movement in direction apex and of Lateral traction to the side walls. This type of instrumentation considered classic or conventional determined an increase in the diameter of the root canal corresponding to the increasing numerical increase of the diameters of the instruments, being that instrumentation realized in apico/coronal sense and in the whole extension of the conduit. In order to facilitate and improve the efficiency of the technique, in 1899 we started talking about mechanical or mechanized instrumentation, which relieved the dentist from working, and a drill was started inside the duct operated with a dental motor. To avoid fractures of the instruments, the number of revolutions was limited to $100 \mathrm{rpm}$. But it was not until the arrival of the racer lime head in 1958, with longitudinal oscillatory movements, and with the contraceptive of Giromatic, in 1964, when the real era began or was the mechanical/ rotational instrumentation of the root canal.

After the appearance of the Giromatic (MicroMega) contraangle, over the last 50years many techniques of mechanical instrumentation have used many flexing movements. Some of them use reciprocating rotations (Giromatic) with a speed of 300rpm, considered the most known mechanical system called Kerr ENdolift. This consisted of a traction movement combined with quarter turn revolutions. While the Endocursor system operated with continuous rotation movements and the IntraEndo system with linear traction movements. However, all these systems were criticized for their ability to model the root canal system due to the constant formation of steps and deviations of the ducts, and to convert the curved ducts to too straight, with the risk involved. In the mid-1980s, a new system emerged, marking the transition to more flexible rotary systems and an increase in its longitudinal torsional capacity, the CanalFinder system: this operated with linear movements of 0.4 to $0.8 \mathrm{~mm}$. This type of instrumentation evolved even more with the advent of a new composite material for titanium nickel files.

\section{Nickel titanium in endodontics: advantages and disad- vantages}

Nickel-titanium alloys were developed in US Navy laboratories in the 1970s. His first application in dentistry, was for the use of orthodontic wires, due to its great resistance to fatigue. Dental alloys range from $56 \%$ nickel to $44 \%$ titanium, as is the case with Endodontics. This together with the technological advance and its subsequent application to rotary systems confers the same elasticity, flexibility and resistance to the plastic deformation and fracture. According to the latest evidence nickel titanium has demonstrated greater flexibility and resistance to fracture by torque compared to stainless steel instruments. The nickel-titanium supposedly also absorbs tensions and resists wear better than stainless steel. Of interest are the special properties provided by this alloy, such as the memory effect, that is, the nickel-titanium returns to its initial shape after deformation and thus shows a super elasticity; So these instruments can not be nor is required, pre-curse. Moreover, nickel - titanium files can deform up to $10 \%$, recovering their initial shape, while those of stainless steel are only possible by $1 \%$. We must not forget how the plastic deformation of an alloy is characterized by its capacity to suffer permanent deformations, without reaching the rupture. This property allows to evaluate the capacity of mechanical work that the material could support, preserving, notwithstanding its physical integrity. Nickel - titanium files are manufactured to be used mechanically rotary as for manual instrumentation, logically changing their design adapted to each use. There may be differences between the two types in the patterns of deterioration (reflected by wear and fracture). Hand instruments give us some tactile feel, which would help us detect weakening or loss of instrument sharpening. On the contrary, rotary machining instruments allow wear and/or fracture without previous warning signs. Given its physical behavior, the alloy and hence the nickel-titanium files present two crystallographic phases. That is to say, each lime, made with this type of alloy, when it is at rest is in the austenite phase, and when it is in rotary movement, it presents/ displays a deformation known like martensite, prone to the fracture, more therefore this type of Limes than those made in stainless steel. As already mentioned, two types of fracture are considered: torsional, in $55 \%$ of the total number of fractures of nickel - titanium files and those of the tensile type, $45 \%$ of them. Despite the above, other complications that can occur when using this type of instrument is the cyclic fatigue of the same. This refers to the dimensional changes that the instrument presents after each time it is used due to the movement of flexion and deflection, or explicitly to the number of rotations to which it has been exposed inside the root canal system, which increases with the Degree of curvature than the present duct.

Another set of factors that may favor fracture of the instruments are: disinfection and sterilization measures and micromotor revolutions, as more important. Disinfection with glutaraldehyde, as with hypochlorite at $2.5 \%$ and up to ten cycles of sterilization, was found to have no influence on the strength characteristics of the limes and therefore did not favor their possible fracture. As for speed, it is not advisable to exceed $350 \mathrm{rpm}$, as this may favor an intrinsic degeneration of the instrument, although in many cases a dependent factor, in which evidence is growing, of the operator and his experience with the system. Another factor to take into account is the power of cut: it was traditionally said that the manual instrument has greater cutting capacity compared to a greater constancy of the rotary, being again the experience a factor difficult to measure in any study that can buy both aspects. 
At present, the design of instruments and materials are finally adapting to the objectives of cleaning and disinfecting the duct and its subsequent filling, not forgetting comfort, speed and safety for professional and patient. Nickel titanium alloys have allowed for new sheet designs, larger sharpened instruments, alternative size systems and the introduction of rotary movements for cleaning and shaping root canals.

\section{Discussion}

Rotary endodontics, with each of its possible systems and technique, as well as the manual procedure, have the same objectives:

a. Remove from the duct system material that is capable of maintaining bacterial growth or degrading into destructive tissue by-products.

b. Disinfection of microorganisms of the root canals before and during conducting the root canal.

c. Design and preparation within each root canal the cavity shape that encourages the most effective, simple and safe sealing and three-dimensional hermetic seal, with the least investment in time, material and possible cost.

d. Establish a conical shape of continuous narrowing.

e. Make the conic preparation exist in multiple planes, not only in those where a geometric cone can be described.

f. Maintain the conduit in an original space situation.

g. Maintain the apical foramen in an original spatial position.

h. Preserve the apical foramen as small as possible.

Although perhaps this can be summarized in the accomplishment of two goals: cleaning and conformation of the conduit, thus ensuring disinfection, hermetism and apical sealing, that allows a later reconstruction with favorable prognosis in the long term. Thus the current evidence shows how manual instrumentation is still the most used, although the disadvantages in terms of lack of flexibility, little conicity and slowness at work.

The emergence of these systems of instrumentation has triggered a true cascade of offers of different systems in the market that provide the professional in endodontics a great variety of products as well as techniques, which with the same principle, differ in technical aspects in terms of characteristics Of the instrument or the type of sealing of the conduit, for example. This same variety generates a problem in deciding which system of work is best for our daily clinical practice, and To question us if there can be a technique, process or material more indicated before certain types of indications or cases.

It is essential to know the advantages and disadvantages described in rotary instrumentation. So as for the advantages, it is possible to be outlined:

i. Reduction in working time.

ii. Better cleaning of the root canal, although some authors do not find statistically different data.

iii. Better and more accurate root canal sealing.

iv. Better biomechanical preparation of the conduit in a smaller portion of exposure time, compared to mechanical instrumentation. v. Better irrigation, thanks to a more conical morphology that allows rotary instrumentation. On the contrary, we find a large number of disadvantages, some of which pose a great risk, closely linked to an incorrect control of the technique:

The highest failure rate is associated with the fracture of the instrument. More than $90 \%$ of nickel-titanium instrument fractures occur while being used in continuous rotation. There is no clear data, whether this fracture rate is greater or not at the beginning of the instrumentation or as the diameter of the duct light increases.

This corresponds to a double causation:

A. error in the manipulation by the operator not respecting the instructions for an appropriate use.

B. fatigue of the material caused by the rapid succession of compressions and extensions of the instrument in a duct of difficult morphology.

Most hand instruments are stainless steel and are designed as bolts, having one or more cutting edges. This is intended to be used for liming, eroding and at the same time forming, in a reciprocating longitudinal movement, the walls of the conduit at a determined length. The problem arises, when they happen to use continuous rotation, as happens in the rotary instrumentation. As it increases the risk that they are screwed or blocked in the walls of the conduit, which inevitably ends in the fracture of the instrument, which together with whether it is an instrument that has already undergone previous wear (imagine a 4-conduit endodontic), Increases the risk of fracture and, worse, in which region of the duct. This was tried to avoid with two fundamental mechanisms: a reduction of the cutting angles of the edges of the instrument and with a cutting edge less pronounced, with a lower angulation. These modifications have often proved to be useless, even without taking into account that they lead to a reduction in cutting efficiency and therefore an increase in the work of the instrument, which goes hand in hand with greater frictional wear and increasing the risk of fracture. To perform a rotary endodontic treatment we need a motor and a system of rotating files for the preparation of each of the root canals.

\section{The engine of endodontics}

These instruments were designed for use through rotational movements clockwise, using electric motors that offer constant speed without oscillation between 150-600rpm. Some also offer even automatic torque control. This peculiarity represents a drastic importance since when the instrument is associated clockwise and for some reason reaches its limit of resistance, which may be predetermined in some devices, this instrument stops automatically. Many of the current engines this rotary movement is reversed counterclockwise, when the pre-set torque is reached which will keep the instrument from leaving the root canal normally. Some apparatuses have torque control devices, preferably automatic, ranging from 0.1 to $10 \mathrm{Nw}$ per centimeter. The different brands or commercial houses of the corresponding rotary system usually offer their own motor, some of them portable and with rechargeable battery, that facilitates and it diminishes the noise pollution and its transport and storage, some of them like:

\section{i. NSK engine}

ii. TCM Endo (Nouvag, Switzerland) 


\author{
iii. EndoPro (Driller, Brazil) \\ iv. EndoPlus (Driller, Brazil) \\ v. Quantec E Endodontic System 8Analitic Sybron, USA) \\ vi. Tri Auto ZX 8Morita, Japan) \\ vii. Tulsa Dental (Dentsply)
}

\section{The lime system}

The systems of files offer a great versatility to the professional, from which they prefer a greater instrumentation to those who prefer a minimum apical deformation, or on the other hand those that need more tapper by the type of ulterior shutter, those that prefer to simplify the technique with Just use two files etc.

What is certain is that each duct is different and each duct marks some needs so it may be more suitable a type of instrumentation and therefore a type of files. All of them share a number of fundamental components: a) Taper/conicity, b) section of the instrument, c) cutting angle, d) instrumentation technique, e) file body

A. Taper: it is the conicity that we give to our behavioral preparation, the better taper a better entrance of the irrigating solution, and therefore better disinfection, better adaptation of the material shutter and less traction of the files, without excesses since An excessive taper can greatly weaken the root.

B. Section of the instrument: we will indicate especially the relationship of the instrument with the dentinal walls, it is usually triradial or birradial being more stable the centering of the file the more supports we have.

C. Cutting angle: gives us the aggressiveness to cut. There are instruments with negative cut, like Profile, whose action is more of wear than of cutting, others like Protaper or Mtwo are active cutting which will remove more amount of dentin in less time, although on the other hand that greater aggressiveness makes them less safe.

D. Instrumentation technique: two different ways can be given in the way that files have to work. Usually it is a cornoapical technique, although we have systems like the Mtwo that works from the first file to length of work. This type of instrumentation is considered the most appropriate because it will favor progressive decontamination and less stress in the file since it does not work in tioda its length.

E. Body of the file: the amount of material that is made will give us greater or less robustness. The following is a list of some of the rotary instrumentation systems available in the market and according to the current evidence are the most used with acceptable long-term results: a) Protaper, b) Profile, K3, Heero642, Lightspeed, SistemaGT, Quantec, PowerR, Flexmaster, RaCe, S-Apex, Endosequence, EndoEZE 8AET), Mtwo, EndoWave, Endo-Express, Navyflex, Liberator, Lightspeedextra, NITI-Tee, Endomagic, PedoWave.

Following a chronological order and current use, we will compare the main systems used and currently part of daily clinical activity:

a) GT 8GT rotatory files system: an old system that was updated to adapt to the new systems: GT rotary files, designed by Stephen Buchanan, prepare the root canals following a crow-down technique similar to the profile files. They feature a U-shaped cross-section with a radial bearing surface that prevents screwing and keeps the instrument centered inside the root canals, preventing the risk of a zip or gap. The tip is conical and inactive, respecting the trajectory of the ducts without risk of transport or false routes. GT files are used in a contra-angle at a constant speed ranging from 150-350RPM.

b) K3: system that evolved to the classics Quantec2000, Quantec SC, Quantec Xl, has a passive tip and the angle of cut is slightly positive while the variable helical angle is $31^{\circ}$ in the tip and $43^{\circ}$ in the rest of the active part, Allowing a waste removal through the space of the striae. The wide radial plane prevents the propagation of cracks and reduces the chances of fracture and deformities of the instrument by torsional stress. One of the advantages of this system can be the reduced handle, presenting files $5 \mathrm{~mm}$ shorter than the others, reducing the size that resides in the handle and not in the active part. Its speed does not exceed 300RPM.

c) Hero micromega with inget: is the conventional Hero system with an innovation: a motor head shorter and therefore versatile. JM Vulcain and P Calas were the creators of the initial system of hero642 that was modified incorporating the novelty of a micromotor that owns a cabezal of smaller size to reach more easily to later sectors. This new system is called INget. Hero files are instruments with three points of support with a triple section 's' to center the file on the Inside the duct and achieve a greater circularity of the same. They have a large central mass of the rod that decreases the risk of fracture. In the longitudinal sense, they have a variable helical angle that limits the interlocking effect of the instrument in the dentine of the duct walls, while facilitating the evacuation of the dentinal remains. The speed of rotation in this case is 360600RPM.

d) Protaper: some d more employees and moreyears in the market. Designed by Clifford Ruddle, Pierre Machtou, Joh West, the main features with their multiple and progressive conicity, a slightly negative cutting angle, a convex triangular cross section, rounded edges with variable piych and a non-cutting inactive tip. This system was initially made up of 6 files, at the end of 2006, a modification of its section reached in some of its files, expanding the system with new files of apical conformation, giving rise to a new generation the system commercialized as Protaper Universal. It seeks to implement longer ducts, with larger apical calibers and less risk of fracture of the instrument. Uses speeds of 150 to 350RPM.

e) Mtwo: it is one of the systems of recent appearance, of which there is a smaller number of scientific evidence. This system, created by Dr. Malagnino, consists of a rotary instrumentation of nickel titanium that brings the novelty of a complete instrumentation of the duct from the entrance of the canal to the apex, from the first file. It is presented as an easy-to-use system and a variable pitch that reduces the risks of fracture, inactive tip, negative cutting angle and cross section in italic 'S' with two active cuts trying to minimize screwing, apical transport and deformations Of the conduit. The Mtwo system is used at about 150-350RPM.

f) Twisted file: This is a new system of nickel titanium files for rotary endodontics created by Dr. Richard Munce and presented by Sybron Endo. The files have a triangular cross-section and their NT wire structure is subjected to a heating-cooling process created by Sybron Endo, which allows a twist of the metal, resulting in a much more flexible file with a very high fracture resistance Impro- 
ved and increased cutting efficiency, which oscillates 3 or 4times higher than other systems. Furthermore, TF files are considered superior because they are not subjected to surface wear in their manufacture which is present in other NiTi systems. Microfractures that would induce a complete fracture in a simpler way are thus avoided. For this rotation system the speed is higher, about 500RPM.

\section{Conclusion}

It is worth mentioning the data tangle of each of the systems, the existence of uncontested evidence and the absence of comparative articles in any of the possible items of Study: ease of use, risk of fracture of the instrument, success of the canal obturation, to give some examples. One of the fundamental objectives in the instrumentation of the root canals is the cleaning or removal of the infection from the inflamed pulp and the conformation of a space for root obturation. These two characteristics are also the most studied and make up the main reason for the use of files or rotary / mechanized instrumentation system. Thus, the first studies about the Mtwo system compare their ability to clean conduits with other systems such as Profile, Hero and K3.

The study by Foschi et al. ${ }^{1}$ Compared the cleaning of the Protaper and Mtwo systems to the electron microscope. It was observed that the most significant differences were not between the two systems, but in the different zones of the ducts. The results were similar, with no significant differences, with a good cleaning in the middle third and coronal in both cases. In the apical third, however, traces of dentin barrel and debris remained in all ducts. In 2005, M. Veltri et al. ${ }^{2}$ Carried out a comparative study between the Mtwo and EndoflareHero Sharper systems, which shows how the dentin removed in this case was similar in both ducts with small differences, although the apical third Hero loses $0.03 \mathrm{~mm}$ more working length compared to Mtwo (losing 0.55) surely for many a nonsignificant difference. Orgaz Uyanik et al tested the Hero Shaper, Protaper and RaCe systems using a computerized tomography system. After the study it was evidenced that Protaper removed much more dentinary barrel than Hero Shaper. Regarding the GT system, it is worth mentioning that it is in good agreement with systems such as K-Files, Lightspeed and the Profile itself, making a better conformation of the duct according to Peters et al.

Any system here treated would be valid for a good cleaning of the duct, although none has been able to eliminate all the detritus. If we ask the question, not easy or even resolved, if rotary cleaning improves the manual, it repeats the tangle of contradictory studies and often poor scientific evidence. According to Clark Dato et al., We can say that, with a progressive instrumentation system and regardless of the system used, there are no detectable differences in the numbers of colony forming units left after using one or the other system. Bacteriafree duct.

We must not forget when selecting a rotary system or another, the preservation of the original shape of the duct after performing the instrumentation. Almost all studies cited previously also studied this characteristic, arriving at the conclusion that in the Hero and Mtwo systems there are no differences in this aspect. Both respect the initial shape of the conduit, because compared to systems such as the K3, the Mtwo system does have advantages and a better behavior, preserving the anatomy of the ducts considerably better as stated in a review Schafer et al. ${ }^{3}$ OA Peters gives us a significant amount of information about the amount of material removed, comparing the Protaper system with the GT, showing how the low aggressive removal preserves the original shape of the duct better and avoids fracture risks. The main drawback of rotational endodontics is fracture. The studies are not relevant in many cases, since if we use new files and a correct technique the possibilities of fracture will be reduced for any system. $^{4-9}$

We must not forget a determining factor or aspect, the speed of preparation of the ducts: it has been considered the main advantage with respect to manual endodontic techniques. AH Gluskin compared the instrumentation with rotating GT files and the Flexofile manual, where it was verified how the rotary system is notably faster, with a preapproval time of $5.9(+/-3)$ minutes, compared to $23.2(+/-9)$ of manual instrumentation. This study is supported by many others which demonstrate the reduction of working time with mechanical instrumentation systems in general.

Regardless of the endodontic system used, manual or rotary, and regardless of the type of file, motor or material forming the file, it is always basic to take into account the complexity of a treatment of ducts by intrinsic variables Tooth, patient characteristics etc.) and extrinsic, operator and auxiliary dexterity or condition of the material. Endodontics requires two characteristics: time and knowledge to know how to respond to the tooth and the instrument.

\section{Acknowledgements}

None.

\section{Conflict of interest}

Author declares that there is no conflict of interest.

\section{References}

1. Foschi F, Nucci C, Montebugnoli L, et al. SEM evaluation of canal Wall dentine following use of Mtwo and Protaper Niti rotatory isntruemnts. Int Endodon. 2004;37(12):832-839.

2. Veltri M, Mollo, Mantovani L, et al. A comparative study of endoflare - Hero Shaper and Mtwo NiTi instruments in the preparation of curved root canals. Int Endodon J. 2005;38(9):610-616.

3. Schafer E, Erler M, Dammaschke T. Comparative study on the shaping ability and cleaning efficiency of rotary Mtwo instruments. Cleaning effectiveness and shaping ability in severely curved root canals of extracted teeth. Int Endodon J. 2006;39(3):206-212.

4. Albuquerque Matos M, De Root i Porta JM, Roig Callon M. Sistema de intrumentación protaper universal. RODE. 2007;5:79.

5. Boada C, Moré A, Rueda LM, Niño J, et al. Comparación in vitro de dos sistemas rotatorios de instrumentación de los conductos radiculares. Endodoncia. 2003;24:3.

6. Facundo M. Limas K3. Características de las limas. RODE. 2005;5:23.

7. Fagondo Morales CM, Contreras Lovera Z, De Ribot Porta J. Sistema Mtwo NiTi: técnica clínica. RODE. 2007;5:25.

8. Luzi A, Almenar garcia A, Corner Navarro. Mtwo: un nuevo sistema rotatorio para la isntrumentación de los conductos radicualres. Endodoncia. 2003;24(3):154-160.

9. Schafer E, Erler M, Dammaschke T. Comparative study on the shaping ability and cleaning efficiency of rotary Mtwo instruments. Part 1. Shaping ability in simulated curved canals. Int Endodon J. 2006;39(3):196-202. 